\title{
Lanthanide-Loaded Liposomes for Multimodality Imaging and Therapy
}

\author{
Sander W. Zielhuis, ${ }^{1}$ Jan-Henry Seppenwoolde, ${ }^{2}$ Vanessa A.P. Mateus, ${ }^{1}$ Chris J.G. Bakker, ${ }^{2}$ \\ Gerard C. Krijger, ${ }^{3}$ Gert Storm, ${ }^{4}$ Bernard A. Zonnenberg, ${ }^{1}$ Alfred D. van het Schip, ${ }^{1}$ \\ Gerben A. Koning, ${ }^{3}$ and Johannes F.W. Nijsen ${ }^{1}$ \\ ${ }^{1}$ Department of Nuclear Medicine, University Medical Center, Utrecht, The Netherlands \\ ${ }^{2}$ Department of Radiology, University Medical Center, Utrecht, The Netherlands \\ ${ }^{3}$ Department of Radiation, Radionuclides and Reactors, Delft University of Technology, Delft, The \\ Netherlands \\ ${ }^{4}$ Department of Pharmaceutics, Utrecht Institute for Pharmaceutical Sciences, Utrecht University, \\ Utrecht, The Netherlands
}

\begin{abstract}
Many advanced molecular imaging agents are currently being investigated preclinically. Especially, liposomes, have proven to be very promising carrier systems for diagnostic agents for use in single-photon emission computed tomography (SPECT) or magnetic resonance imaging (MRI), as well as for therapeutic agents to treat diseases such as cancer. In this study, nanosized liposomes were designed and labeled with the radionuclides, holmium-166 (both a beta- and gamma-emitter and also highly paramagnetic) or technetium-99m, and coloaded with paramagnetic gadolinium allowing multimodality SPECT and MR imaging and radionuclide therapy with one single agent. Methods: Diethylenetriaminepentaacetic acid bisoctadecylamide (an amphiphilic molecule with a chelating group suitable for labeling with radionuclides) and gadoliniumacetylacetonate (GdAcAc) (a small lipophilic paramagnetic molecule) were incorporated in liposomes. The liposomes were characterized by measuring their mean size and size distribution, gadolinium content, and radiochemical stability after incubation in human serum at $37^{\circ} \mathrm{C}$. The MRI properties (in vitro) were determined by use of relaxivity measurements at 1.5 and 3.0 Tesla in order to evaluate their potency as imaging agents. Results: The liposomes were successfully labeled with holmium-166, resulting in a high labeling efficiency $(95 \% \pm 1 \%)$ and radiochemical stability $(>98 \%$ after 48 hours of incubation), and coloaded with GdAcAc. Labeling of liposomes with technetium-99m was somewhat less efficient $(85 \% \pm 2 \%)$, although their radiochemical stability was sufficient $(95 \% \pm 1 \%$ after 6 hours of incubation). MRI measurements showed that the incorporation of GdAcAc had a strong effect on the MRI relaxivity. Conclusions: The synthesized liposomes allow for multimodality imaging and therapy, which makes these new agents highly attractive for future applications.
\end{abstract}

Key words: liposomes, molecular imaging, MRI, gadolinium, holmium, SPECT, technetium

Address reprint requests to: Johannes F.W. Nijsen; Department of Nuclear Medicine, University Medical Center Utrecht; Heidelberglaan 100, Room number: E02.222, 3584 CX Utrecht, The Netherlands; Tel.: (31) 30 2506295; Fax: (31) 302542531

E-mail: F.Nijsen@azu.nl

\section{INTRODUCTION}

It is the ultimate goal of diagnostic medical imaging to achieve highly specific visualization of fundamental biological processes based on morphologic, physiologic, molecular, and genetic 
markers of diseases such as cancer. ${ }^{1}$ This goal is believed to be realized through the development of multimodality imaging facilities and highly specific diagnostic agents. For an optimal diagnostic procedure and subsequent treatment, it is furthermore important to accurately pinpoint anatomically the position of the imaging agent. ${ }^{2}$ This can be accomplished through multimodality imaging by use of both single-photon emission computed tomography (SPECT) and magnetic resonance imaging (MRI). ${ }^{3}$ Merging images of SPECT and MRI combines the high sensitivity of SPECT with the anatomical information of MRI. ${ }^{2}$ However, at this moment, no imaging agents have been designed that can be visualized with both SPECT and MRI. In order to achieve multimodality imaging with a single agent, both radionuclides and MRI contrast agents have to be combined in one targeted vehicle. Furthermore, if multimodality imaging agents are also loaded with therapeutic agents, targeted therapy of, for example, cancer, can be subsequently visualized. ${ }^{4}$ Specific imaging and targeted therapy can be achieved by using liposomes, which can be targeted to tumor sites by the coupling of socalled homing devices (e.g., [fragments] of antibodies, to the surface of liposomes). ${ }^{5-8}$ These vesicles $(\sim 50-500 \mathrm{~nm})$ consist of an aqueous space surrounded by a lipid bilayer. Liposomes can be labeled with radionuclides, such as indium- $111^{9}$ and technetium- $99 \mathrm{~m},{ }^{10}$ by linking the radiolabel to the lipid bilayer, either directly or through the use of a chelator. These liposomes have been applied for the scintigraphic detection of lymph nodes ${ }^{10}$ or inflammation. ${ }^{11}$ For radiotherapeutic purposes, alpha- ${ }^{12}$ and beta-emitters $^{13}$ have been incorporated in liposomes.

Liposomes have also been used to enhance (tumor) diagnosis with MRI by loading them with highly paramagnetic elements, with gadolinium as the most pronounced example. ${ }^{4}$ These liposomes were successful in the detection of lymph nodes ${ }^{14}$ and the visualization of prostate adenocarcinoma in animal models. ${ }^{15}$ Loading of liposomes with gadolinium can be achieved by incorporating amphiphilic gadolinium derivates into the liposomal bilayers ${ }^{4}$ to obtain an enhanced MR contrast. This contrast generally results from the shortening of the longitudinal relaxation time $\left(\mathrm{T}_{1}\right)$ of the MRI signal by the local magnetic field inhomogeneities created by the loaded liposomes. It would be a great advantage if liposomes can be labeled with both paramagnetic elements and radionuclides. This combined loading would al- low for imaging and therapy by use of one single agent, which can be visualized by two imaging modalities. It is, furthermore, a challenge to improve the relaxation properties of the liposomes, allowing for a more sensitive detection with MRI.

This paper describes the preparation and characterization of liposomes loaded with both radionuclide and paramagnetic compound. Liposomes were labeled with holmium-166, a therapeutic radionuclide that has been thoroughly investigated by our research group because of its unique therapeutic and diagnostic characteristics. ${ }^{16-18}$ Holmium can be easily neutron-activated to the radioactive isotope holmium-166 ( $0.1 \%$ of the total amount of holmium), a betaand gamma-emitter with a logistically favorable half-life (26.8 hours). Holmium, whether radioactive or not, can also be visualized by MRI. ${ }^{17,18}$ It should be realized that because of the different size (as compared to holmium-loaded microspheres that can be used for treatment of liver malignancies ${ }^{16,17}$ ), the liposomes are expected to act as a $T_{1}$ shortening agent, whereas the large microspheres act as a $\mathrm{T}_{2}$ agent. ${ }^{17}$ To enable scintigraphic detection, technetium labeled liposomes were prepared. The liposomes were made paramagnetic by coincorporation of gadolinium, utilizing a novel procedure based on a small lipophilic gadolinium complex.

Liposomes were characterized by measuring their radiochemical stability and MRI properties in order to evaluate their potential usefulness as new multimodal diagnostic and therapeutic agents.

\section{MATERIALS AND METHODS}

\section{Materials}

All chemicals were commercially available and used as obtained. Acetylacetone, 2,4-pentanedione (AcAc; > 99\%), chloroform $\left(\mathrm{CHCl}_{3}\right.$; highperformance liquid chromatography [HPLC] grade), cholesterol (Chol; $>99 \%)$ and ammonium hydroxide $\left(\mathrm{NH}_{4} \mathrm{OH} ; 29.3 \%\right.$ in water) were supplied by Sigma Aldrich (Steinheim, Germany). Holmium (III) chloride hexahydrate $\left(\mathrm{HoCl}_{3} \cdot 6 \mathrm{H}_{2} \mathrm{O} ; 99.9 \%\right)$ and gadolinium (III) chloride hexahydrate $\left(\mathrm{GdCl}_{3} \cdot 6 \mathrm{H}_{2} \mathrm{O} ; 99.9 \%\right)$ were obtained from Phase Separations BV (Waddinxveen, The Netherlands). 1,2-dipalmitoyl-sn-glycero-3-phosphocholine (DPPC) and 
1,2-dipalmitoyl-sn-glycero-3-phospho-ethanolamine- $N$-[methoxy(polyethylene glycol)-2000] (PEG-DSPE) were obtained from Lipoid (Ludwigshafen, Germany). Diethylenetriaminepentaacetic acid bisoctadecylamide (DTPA-lipid) was obtained from Gateway Chemical Technology Inc (St. Louis, MO). Manganese (II) chloride tetrahydrate $\left(\mathrm{MnCl}_{2} \cdot 4 \mathrm{H}_{2} \mathrm{O} ; 99.9 \%\right)$, hydrochloric acid ( $\mathrm{HCl} ; 37 \%)$, and methanol $\left(\mathrm{CH}_{3} \mathrm{OH}\right.$; HPLC-grade) were obtained from Merck (Darmstadt, Germany). Stannous chloride dihydrate $\left(\mathrm{SnCl}_{2} \cdot 2 \mathrm{H}_{2} \mathrm{O}\right.$; 99.9\%) was purchased from Riedel-de Haën, (Seelze, Germany).

\section{Preparation of Liposomes}

Liposomes were prepared by the conventional thin-film hydration technique, as described previously, ${ }^{19}$ and consisted of DPPC, Chol, and PEG-DSPE in a molar ratio of 1.85:1:0.15. Labeling of liposomes with the radionuclides holmium-166 and technetium-99m was achieved by use of the amphiphilic molecule, DTPA-lipid, which was incorporated into the liposomal bilayer. This molecule consists of a chelating group, to which two fatty acids $(\mathrm{C} 18)$ are at- tached. The paramagnetic element, gadolinium, was incorporated into the bilayer of the liposomes in a new way using its acetylacetonate complex (GdAcAc), a small lipophilic molecule. GdAcAc was prepared, as described previously, for HoAcAc. ${ }^{16}$ Acetylacetone (180 g) was dissolved in water $(1080 \mathrm{~g})$. The $\mathrm{pH}$ of this solution was adjusted to 8.50, with an aqueous solution of ammonium hydroxide. Gadolinium chloride (10 g dissolved in $30 \mathrm{~mL}$ of water) was added to this solution. After 15 hours of incubation at room temperature, the formed GdAcAc crystals were collected by centrifugation and washed with water.

Four types of liposomes were prepared: liposomes loaded with $20 \mathrm{~mol} \%$ DTPA-lipid (type A), $20 \mathrm{~mol} \%$ GdAcAc (Type B), a combination of 10 mol\% of GdAcAc and 10 mol\% DTPA-lipid (type C), or a combination of $20 \mathrm{~mol} \%$ of GdAcAc and 3 mol\% DTPA-lipid (type D). A schematic representation of the various liposome types is given in Figure 1. DTPA-lipid and GdAcAc were included in the liposomes by replacing equal molar amounts of DPPC. The lipid mixture (a total amount of $300 \mu \mathrm{mol}$ ) was dissolved in a mixture of $5 \mathrm{~mL}$ of chloroform and $2.5 \mathrm{~mL}$ of methanol

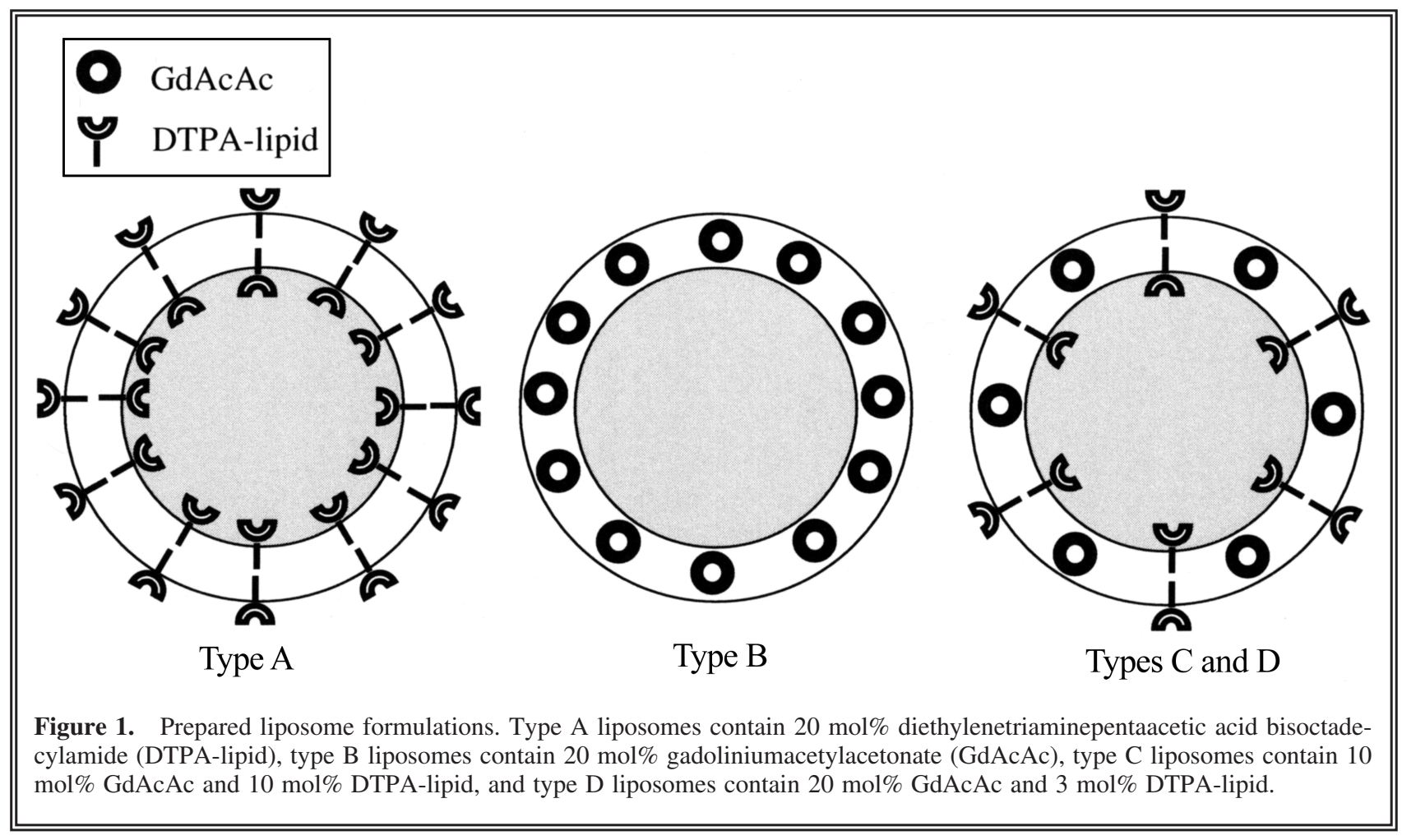


and evaporated to dryness by rotary evaporation under vacuum. The resulting lipid film was further dried under a stream of nitrogen and subsequently hydrated in $6 \mathrm{~mL}$ of an ammonium acetate buffer $(100 \mathrm{mM})$, with a $\mathrm{pH}$ of 5.0. The resulting lipid dispersion was extruded, using polycarbonate membrane filters (Poretics Corp.; Livermore, CA) with a pore diameter of 600,400 , 200 and $100 \mathrm{~nm}$ for 2, 2, 6, and 8 times, respectively. After preparation, the types A and C liposomes were labeled with holmium-166, and the type D liposomes were labeled with technetium99m. All liposomes were coated with polyethylene glycol (by use of PEG-DSPE), as it has been reported that this polymer has a positive influence on the in vivo circulation time and the MRI characteristics of the liposomes. ${ }^{4}$ Liposome batches were prepared in duplicate.

\section{Liposome Characterization}

The phospholipid content was determined with a phosphate assay, according to Rouser et al., in order to determine the total lipid concentration after extrusion. ${ }^{20}$ The average size and size distribution (polydispersity index) of the liposomes were determined by dynamic light scattering using a Malvern ALV CGS-3 (Malvern Instruments Ltd.; Worcestershire, United Kingdom). The polydispersity index is a measure for variation in particle size within a liposome population, and varies from 0 (complete monodispersity) to 1 (large variations in particle size), and was calculated according to the method of Zhao et al. ${ }^{21}$ The amount of GdAcAc in the liposomes was measured by inductively coupled plasma optical emission spectrometry (ICPOES). Liposome samples were lysed in a $1 \%$ Triton (w/v) solution. Samples were next introduced in an Optima 4300 CV (PerkinElmer; Norwalk, CT) ICP-OES spectrometer. Total gadolinium content was determined by detecting emission at $336.223 \mathrm{~nm}$ and related to a standard calibration curve, measured between 0 and $20 \mathrm{mg} / \mathrm{L}$. The stability of GdAcAc-loaded liposomes was determined by dialyzation of 0.5-mL liposomes $(25 \mu \mathrm{mol})$, using Slide-ALyzer dialysis cassettes with a molecular weight cut-off of 10,000 (Pierce; Rockford, IL) at $37^{\circ} \mathrm{C}$ against $25 \mu \mathrm{L}$ of $10 \mathrm{mM}$ phosphatebuffered saline (PBS; pH 7.4). After 48 hours of dialyzation, the release of gadolinium in the buffer was measured with ICP-OES.

\section{Radiolabeling of Liposomes}

Holmium-166 was obtained by neutron irradiation of $50 \mu \mathrm{mol}$ holmiumchloride (packed in polyethylene vials) for 4 hours with thermal neutron flux of $5 \times 10^{12} \mathrm{~cm}^{-2} \mathrm{~s}^{-1}$ at the Reactor Institute in Delft (Department of Radiation, Radionuclides and Reactor; Delft University of Technology, Delft, The Netherlands). After neutron activation, holmiumchloride was dissolved in $0.5 \mathrm{~mL}$ of an ammonium acetate buffer with a $\mathrm{pH}$ of $5.0(100 \mathrm{mM})$. Types $\mathrm{A}$ and $\mathrm{C}$ liposomes were labeled with holmium-166 (37 and 18.5 $\mathrm{MBq}$, respectively). Therefore, the calculated amount of holmium, which is $50 \%$ of the total molar amount of DTPA-lipid (as only half of the DTPA-lipid is available for labeling on the outer leaflet; see Fig. 1), was subsequently added to 0.5 $\mathrm{mL}$ of liposomes. The solution was incubated for 15 minutes at room temperature.

Type D liposomes were labeled with technetium-99m. Therefore, $50 \mathrm{mg} \mathrm{SnCl} 2 \cdot 2 \mathrm{H}_{2} \mathrm{O}$ was dissolved in $50 \mathrm{~mL} 0.1 \mathrm{M} \mathrm{HCl}$, and this solution was subsequently flushed with a stream of nitrogen for 15 minutes. From this solution, $15 \mu \mathrm{L}$ was added to $0.5 \mathrm{~mL}$ of liposomes, followed by the addition of $50 \mathrm{MBq}$ technetium-99m-pertechnetate (obtained from a molybdenum-99/technetium-99m generator [Mallinckrodt; Petten, The Netherlands]) in $0.1 \mathrm{~mL}$ of saline. The solution was incubated for 15 minutes at room temperature.

The labeling efficiency of both holmium-166 and technetium-99m (fraction radionuclide bound to liposomes vs. free fraction) was determined in the liposome fraction after separation on a PD-10 desalting column (Amersham Biosciences; Uppsala, Sweden), and the radioactivity of both the liposomes and the desalting column was measured with a VDC-404 dose calibrator (Veenstra Instruments; Joure, The Netherlands).

The radiochemical stability of radionuclide-labeled liposomes was determined after incubation of $0.5 \mathrm{~mL}$ of liposomes for, respectively, 48 hours (for holmium-166) and 6 hours (for technetium$99 \mathrm{~m}$ ) in $5 \mathrm{~mL}$ of human serum at $37^{\circ} \mathrm{C}$. The stability was determined in the same way as described above for the labeling efficiency.

\section{MRI Phantoms}

The relaxation properties that describe the influence of the liposomes on the MRI signal are the 
longitudinal and transverse relaxivity, $r_{1}$ and $r_{2}$, respectively. The relation between the resultant relaxation $R_{i, e f f}$, [ $s^{-1}$, the concentration of the liposomes $\mathrm{c}[\mathrm{mM}]$, and the relaxivity $\mathrm{r}_{\mathrm{i}}\left(\mathrm{s}^{-1}\right.$ $\mathrm{mM}^{-1}$ ] is given by $\mathrm{R}_{\mathrm{i}, \text { eff }}=\mathrm{R}_{\mathrm{i}, 0}+\mathrm{r}_{\mathrm{i}}[\mathrm{c}]$, with $\mathrm{i}=$ 1,2 and $R_{i}=1 / T_{i}$ using 1,2 for the longitudinal and transverse relaxation, respectively. The effects of the liposomes on the MR relaxation rates (i.e., the relaxivities $r_{1}$ and $r_{2}$ ) were investigated in phantoms. Polystyrene test tubes with a volume of $5 \mathrm{~mL}$ were prepared by diluting 50-250 $\mu \mathrm{L}$ liposomes with an ammonium acetate buffer $(100 \mathrm{mM})$ with a $\mathrm{pH}$ of 5.0 containing $19.2 \mathrm{mg}$ $\mathrm{MnCl}_{2} / 1$. The end volume was adjusted to 5.0 $\mathrm{mL}$. The spiking of the buffer with manganese chloride was done to adjust the relaxation properties of background fluid in the phantoms to make them comparable to human tissue $\left(\mathrm{T}_{1} / \mathrm{T}_{2} \approx\right.$ $1000 / 100$ milliseconds at $1.5 \mathrm{~T}$ ). Consequently, the resulting concentration of the liposomes (expressed as the total amount of lipid) ranged between 0.5 and $2.5 \mathrm{mM}$.

In order to compare the relaxation properties of GdAcAc-loaded liposomes with liposomes that were loaded with gadolinium by use of amphiphilic gadolinium derivates ${ }^{4}$ (the conventional way to prepare paramagnetic liposomes), type A liposomes were also labeled with gadolinium by using the DTPA-lipid. Labeling of liposomes with gadolinium was done in the same way as described above for holmium-166, using the same molar amounts of gadoliniumchloride.

\section{Relaxation Measurements}

Experiments were done on 1.5 and 3.0 Tesla clinical MRI scanners (Achieva; Philips Medical Systems, Best, The Netherlands), using a birdcage receive coil operating in quadrature mode. The tubes were put in a rectangular grid in foam and positioned in the isocenter of the magnet with their long axis oriented parallel to the main magnetic field. A single transverse slice of $10 \mathrm{~mm}$ (perpendicular to the long axis of the tubes) was measured in the middle of the tubes. To measure the $T_{1}$ and $T_{2}$ relaxation for each of the tubes, a multispin echo, combined with an inversion recovery experiment ${ }^{22}$ was performed with the following parameters: field of view (FOV) $230 \times 172 \mathrm{~mm}$, matrix (MTX) $192 \times 135$, echo-time (TE) $8 \times 20$ milliseconds, flip 90, 1 signal average, duration 332 seconds. With a routine implemented in the scanners' software, ${ }^{22} \mathrm{~T}_{1}$ and $\mathrm{T}_{2}$ maps were reconstructed. Then, a circular region of interest was placed on each tube to calculate the av- erage $T_{1}$ and $T_{2}$ values for each concentration. To calculate the $r_{1}$ and $r_{2}$ relaxivities (linear concentration dependence of the relaxation rates) for each type of the liposomes, relaxation rates $\mathrm{R}_{1}\left(=1 / \mathrm{T}_{1}\right)$ and $\mathrm{R}_{2}\left(=1 / \mathrm{T}_{2}\right)$ were plotted against concentration and followed by linear least square fitting.

\section{RESULTS AND DISCUSSION}

\section{Preparation of Liposomes and Characterization}

Table 1 summarizes the characteristics of the prepared liposomes. PEG was included in the liposome composition to obtain so-called long-circulating liposomes, which have the ability to extravasate at solid tumor sites after intravenous administration. ${ }^{23}$ The liposomes had a mean size of approximately $130 \mathrm{~nm}$ and a low polydispersity index $(<0.07)$, indicating that size variation of the liposomes is very small. ${ }^{21}$ The produced liposomes have an average size, favorable for extravasation through "leaky" tumor vasculature in tumors. ${ }^{24}$ ICP-OES showed that the initial amount of GdAcAc used for preparation of liposomes was comparable to the amount of GdAcAc loaded into the extruded liposomes (difference $<10 \%$ ), indicating an incorporation efficiency of approximately $100 \%$. Dialysis of GdAcAc-loaded liposomes (types B, C, and D) for 48 hours against PBS showed that maximal $4 \%$ of the total amount of gadolinium in the liposomes had been released. This low release demonstrated that the GdAcAc-loading remains stably associated with the liposomes.

\section{Labeling Efficiency and Radiochemical Stability of the Liposomes}

Table 2 shows the labeling efficiency and radiochemical stability of the liposomes. The results

\begin{tabular}{|c|c|c|c|}
\hline \multirow{2}{*}{$\begin{array}{l}\text { Liposome } \\
\text { type }\end{array}$} & \multicolumn{2}{|c|}{ Loading (mol \%) } & \multirow[b]{2}{*}{ Size $(\mathrm{nm})$} \\
\hline & $G d A c A c$ & DTPA-lipid & \\
\hline A & 0 & 20 & $123 \pm 10$ \\
\hline B & 20 & 0 & $138 \pm 4$ \\
\hline $\mathrm{C}$ & 10 & 10 & $133 \pm 6$ \\
\hline $\mathrm{D}$ & 20 & 3 & $125 \pm 4$ \\
\hline
\end{tabular}




\begin{tabular}{|c|c|c|c|c|c|}
\hline \multirow{2}{*}{$\begin{array}{l}\text { Liposome } \\
\text { type }\end{array}$} & \multicolumn{2}{|c|}{ Loading (mol \%) } & \multirow[b]{2}{*}{ Radionuclide } & \multirow{2}{*}{$\begin{array}{c}\text { Labeling } \\
\text { efficiency (\%) }\end{array}$} & \multirow[b]{2}{*}{ Stability $(\%)$} \\
\hline & $G d A c A c$ & DTPA-lipid & & & \\
\hline A & 0 & 20 & ${ }^{166} \mathrm{Ho}$ & $95 \pm 1$ & $>98$ \\
\hline $\mathrm{C}$ & 10 & 10 & ${ }^{166} \mathrm{Ho}$ & $95 \pm 1$ & $>98$ \\
\hline D & 20 & 3 & ${ }^{99 \mathrm{~m}} \mathrm{Tc}$ & $85 \pm 2$ & $95 \pm 1$ \\
\hline
\end{tabular}

from the phosphate assay were used to calculate the amount of DTPA-lipid in the liposomes. It was assumed that $50 \%$ of these DTPA groups were available for labeling with holmium-166, in view of the fact that approximately $50 \%$ of the DTPA-lipid is located at the inner leaflet of the liposomal bilayer and is, therefore, not accessible for labeling (see Fig. 1). Liposomes (type A) that were labeled with holmium-166 demonstrated a high labeling efficiency $(95 \% \pm 1 \%)$ and high radiochemical stability in human serum (>98\%). GdAcAc had no influence on the labeling of type $\mathrm{C}$ liposomes with holmium-166; labeling efficiency $(95 \% \pm 1 \%)$ and radiochemical stability $(>98 \%)$ did not differ from liposomes without GdAcAc (type A). This implies that during the production of liposomes, no $\mathrm{Gd}^{3+}$ ions (originating from $\mathrm{GdAcAc}$ ) are captured by the DTPA-lipid, leaving these chelating groups available for labeling with $\mathrm{Ho}^{3+}$.

Labeling of type D liposomes with technetium$99 \mathrm{~m}$ resulted in a somewhat lower efficiency of $85 \% \pm 2 \%$, although their radiochemical stability in human serum was high, namely $95 \% \pm 1 \%$. This implicates that before administration of liposomes to a patient free technetium-99mpertechnetate has to be removed (e.g., with a PD10 desalting column).

In this study, low amounts of radioactivity were used in order to evaluate the feasibility of the radiolabeling approach. For diagnostic or even therapeutic application, a higher specific activity of the liposomes is required. Assuming that, for example, $1 \mathrm{~mL}$ of type $\mathrm{C}$ liposomes (corresponding with an attainable amount of $2.5 \mu \mathrm{mol}$ DTPA-lipid) is used for administration to patients, an amount of $\sim 400 \mu \mathrm{g}$ of holmium can be bound to the liposomes. Irradiation of this amount of holmium in a nuclear reactor with a thermal neutron flux of $5 \times 10^{14} \mathrm{~cm}^{-2} \cdot \mathrm{s}^{-1}$ for 10 hours results in an activity of $10.6 \mathrm{GBq}$ (end of bombardment). This amount of radioactivity is suit- able for therapeutic applications and for nuclear imaging. ${ }^{16}$

\section{MR Relaxation Properties of the Liposomes}

It is generally known that the presence of paramagnetic material affects the relaxation behavior of the surrounding protons. In this study, we used an experimental method to investigate the relaxation effect of paramagnetic liposomes. The results of the relaxation measurements are shown in Figure 2. In general, the relative relaxivities measured at $1.5 \mathrm{~T}$ display a similar dependence of the various designs of the liposomes as those measured at 3.0 T. In most cases, the relaxivities at $1.5 \mathrm{~T}$ are significantly higher in comparison to $3.0 \mathrm{~T}$, given the exceptions for the cases with the presence of holmium, in which the $\mathrm{r}_{2}$ relaxivities are significantly higher at $3.0 \mathrm{~T}$, most probably owing to an increased susceptibility effect. Taking the gadolinium-labeled DTPA-lipid liposomes (type A) as a reference point (the "conventional way" to design paramagnetic liposomes), this figure shows that the relaxivity of the holmium-labeled DTPA-lipid liposomes (type A) is considerably less, as might be expected from the lower magnetic susceptibility of holmium versus gadolinium. ${ }^{25}$ The relaxivity of GdAcAc-loaded liposomes (type B) is substantially higher than the gadolinium-labeled DTPAlipid liposomes (type A). This is owing to the higher amount of paramagnetic material (gadolinium) per liposome, whereas normally, half of the DTPA-lipid is located at the inside of the liposome and can, therefore, not be labeled with gadolinium (see Fig. 1). The introduction of a small amount of DTPA-lipid (3 mol\%) into the GdAcAc-loaded liposomes (type D) slightly decreased the relaxivities. Trading half the GdAcAc for the incorporation of more DTPAlipid (10 mol\% GdAcAc and $10 \mathrm{~mol} \%$ DTPAlipid, type $\mathrm{C}$ ) resulted in relaxivities approxi- 


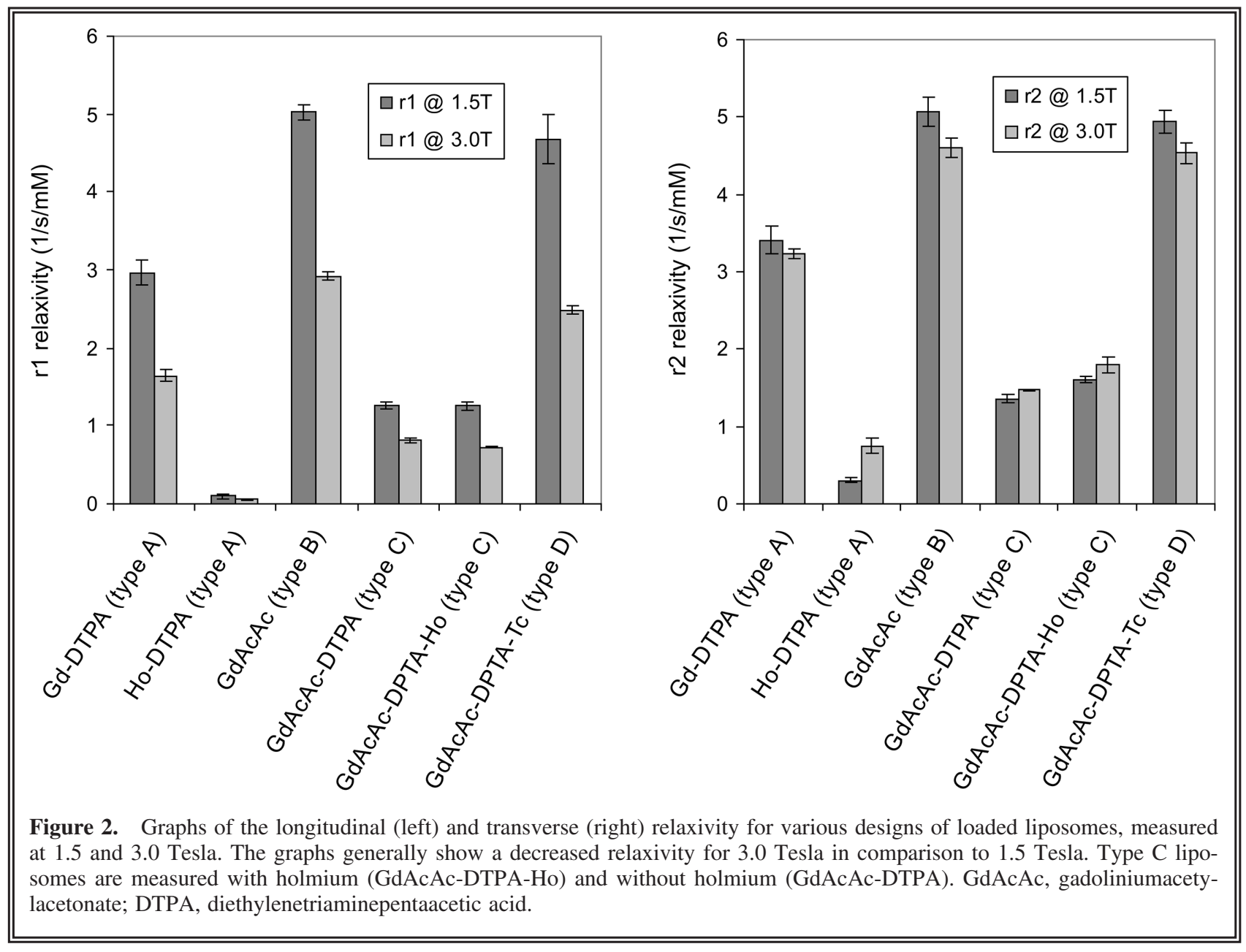

mately half of that of the Gd-labeled DTPA-lipid liposomes. The presence of holmium bound to the DTPA-groups on the surface of the type C liposomes slightly changed the relaxivities, in particular the ratio $r_{2} / r_{1}$, which indicates an increased susceptibility effect, originating from increased magnetic field gradients around the liposomes. ${ }^{26}$

In general terms, the results indicate that the relaxivity of liposomes can be significantly enhanced by the increased incorporation of gadolinium as a small (AcAc) complex inside the liposome bilayer, in comparison to the conventional loading of liposomes with amphiphilic gadolinium derivates. The incorporation of DTPA-lipid reduced this relaxivity somewhat but enables the use of multiple modalities (labeling with technetium-99m) for diagnosis and therapy (labeling with holmium-166).

\section{CONCLUSIONS}

In conclusion, this study demonstrates that it is possible to prepare liposomes with a high GdAcAc loading, which can be labeled afterward with radionuclides, such as holmium-166 and technetium$99 \mathrm{~m}$. These novel liposomes have a high radiochemical stability and are highly paramagnetic, allowing for multimodality imaging and therapy with one single agent. These results represent a significant step forward in the direction of future multimodality imaging and therapy applications.

\section{ACKNOWLEDGMENTS}

This research was supported by the Dutch Technology Foundation STW (UGP.6533), applied 
science division of The Netherlands Organization for Scientific Research, and the technology program of the Ministry of Economic Affairs, Utrecht, The Netherlands.

\section{REFERENCES}

1. Sullivan DC, Kelloff G. Seeing into cells: The promise of in vivo molecular imaging in oncology. EMBO Rep 2005;6:292.

2. Maza S, Taupitz M, Wegner T, et al. Precise localisation of a sentinel lymph node in a rare drainage region with SPECT/MRI using interstitial injection of ${ }^{99 \mathrm{~m} T \mathrm{c}-}$ nanocolloid and superparamagnetic iron oxide. Eur $J$ Nucl Med Mol Imag 2005;32:250.

3. Yang D, Han L, Kundra V. Exogenous gene expression in tumors: Noninvasive quantification with functional and anatomic imaging in a mouse model. Radiology 2005;235:950.

4. Zielhuis SW, Nijsen JFW, Seppenwoolde JH, et al. Lanthanide bearing microparticulate systems for multimodality imaging and targeted therapy of cancer. Curr Med Chem Anti-cancer Agents 2005;5:303.

5. Eliaz RE, Nir S, Marty C, et al. Determination and modeling of kinetics of cancer cell killing by doxorubicin and doxorubicin encapsulated in targeted liposomes. Cancer Res 2004;64:711.

6. Peer D, Margalit R. Loading mitomycin C inside long circulating hyaluronan targeted nanoliposomes increases its antitumor activity in three mice tumor models. Int J Cancer 2004;108:780.

7. Gabizon A, Horowitz AT, Goren D, et al. In vivo fate of folate-targeted polyethylene-glycol liposomes in tumor-bearing mice. Clin Cancer Res 2003;9:6551.

8. Mulder WJ, Strijkers GJ, Habets JW, et al. MR molecular imaging and fluorescence microscopy for identification of activated tumor endothelium using a bimodal lipidic nanoparticle. FASEB J 2005;19:2008.

9. Harrington KJ, Mohammadtaghi S, Uster PS, et al. Effective targeting of solid tumors in patients with locally advanced cancers by radiolabeled pegylated liposomes. Clin Cancer Res 2001;7:243.

10. Phillips WT, Andrews T, Liu H, et al. Evaluation of $[(99 \mathrm{~m}) \mathrm{Tc}]$ liposomes as lymphoscintigraphic agents: Comparison with [(99m)Tc] sulfur colloid and [(99m)Tc] human serum albumin. Nucl Med Biol 2001;28:435.

11. Laverman P, Boerman OC, Oyen WJG, et al. Liposomes for scintigraphic detection of infection and inflammation. Adv Drug Del Rev 1999;37:225.

12. Sofou S, Thomas JL, Lin HY, et al. Engineered liposomes for potential alpha-particle therapy of metastatic cancer. J Nucl Med 2004;45:253.
13. Hafeli U, Tiefenauer LX, Schbiger PA, et al. A lipophilic complex with $186 \mathrm{Re} / 188 \mathrm{Re}$ incorporated in liposomes suitable for radiotherapy. Int J Rad Appl Instrum B 1991;18:449.

14. Misselwitz B, Sachse A. Interstitial MR lymphography using Gd-carrying liposomes. Acta Radiol Suppl 1997;412:51.

15. Luciani A, Olivier JC, Clement O, et al. Glucose-receptor MR imaging of tumors: Study in mice with PEGylated paramagnetic niosomes. Radiology 2004; 231:135.

16. Nijsen JFW, Zonnenberg BA, Woittiez JR, et al. Holmium-166 poly lactic acid microspheres applicable for intra-arterial radionuclide therapy of hepatic malignancies: Effects of preparation and neutron activation techniques. Eur J Nucl Med 1999;26: 699.

17. Nijsen JFW, Seppenwoolde JH, Havenith T, et al. Liver tumors: MR imaging of radioactive holmium microspheres-phantom and rabbit study. Radiology 2004; 231:491.

18. Seppenwoolde JH, Nijsen JFW, Bartels LW, et al. Internal radiation therapy of liver tumors: Qualitative and quantitative magnetic resonance imaging of the biodistribution of holmium-loaded microspheres in animal models. Magn Reson Med 2004;53:76.

19. Koning GA, Fretz MM, Woroniecka U, et al. Targeting liposomes to tumor endothelial cells for neutron capture therapy. Appl Radiat Isot 2004;61:963.

20. Rouser G, Fkeischer S, Yamamoto A. Two dimensional then layer chromatographic separation of polar lipids and determination of phospholipids by phosphorus analysis of spots. Lipids 1970;5:494.

21. Zhao Y, Chen W, Hair D, et al. Light scattering studies of stereocomplex formation of stereoregular poly(methyl methacrylate) in solutions. Eur Polym $J$ 2005;41:447.

22. In den Kleef JJ, Cuppen JJ. RLSQ: T1, T2, and rho calculations, combining ratios, and least squares. Magn Reson Med 1987;5:513.

23. Storm G, Crommelin DJA. Liposomes: Quo vadis? Pharm SciTechnol To 1998;1:19.

24. Charrois GJ, Allen TM. Rate of biodistribution of STEALTH liposomes to tumor and skin: Influence of liposome diameter and implications for toxicity and therapeutic activity. Biochim Biophys Acta 2003;1609: 102.

25. Handbook of Chemistry and Physics, 84th ed. Boca Raton, FL: CRC Press, 2003.

26. Fossheim SL, Fahlvik AK, Klaveness J, et al. Paramagnetic liposomes as MRI contrast agents: Influence of liposomal physicochemical properties on the in vitro relaxivity. Magn Reson Imag 1999;17:83. 